\title{
Segmentierungsbasierte Bewegungsschätzung in Ultraschallbildern
}

\author{
Reise, F.: Rothlübbers, C.; Orglmeister, R. \\ Technische Universität Berlin \\ Institut für Elektronik und Lichttechnik. EN 3 \\ Einsteinufer 17 \\ 10587 Berlin
}

\begin{abstract}
Ein neuer Ansatz zur Verbesserung der Bewegungsschätzung in Ultraschallbildern des menschlichen Herzens wird vorgestellt. Der Ansatz nutzt den Optischen Fluß zur Bewegungsschätzung und bestimmt relevante Bildbereiche über eine Segmentierung auf Basis der Skalierungs-Index-Methode. Vergleiche der Ergebnisse dieses Ansatzes mit den Ergebnissen konventioneller Ansätze zeigen eine deutliche Verbesserung der Bildqualität und ermöglichen so eine bessere Bewertung der Bewegungen des untersuchten Ultraschallbildes des menschlichen Herzens.
\end{abstract}

\section{OPTISCHER FLUSS}

In Analogie zur Strömungslehre findet bei der Untersuchung von Bildfolgen eine ,Strömung" von Grauwerten statt. Diese Grauwertänderungen erfolgen infolge der Abbildung tatsächlicher Bewegungen realer Objekte in der Bildebene. Die Annahme, daß Grauwerte weder entstehen noch verschwinden können, läßt einen unmittelbaren Zusammenhang zwischen den Bewegungen und der zeitlichen Änderung der Grauwerte zu. Dies führt zur Definition einer Kontinuitätsgleichung:

$$
d I(x, y, t)=\frac{\partial I}{\partial x} d x+\frac{\partial I}{\partial y} d y+\frac{\partial I}{\partial t} d t=0
$$

Der Grauwert $l(x, y, t)$ ist eine Funktion der örtlichen Koordinaten $x, y$ und der zeitlichen Koordinate $t$. Zur Lösung dieses unterbestimmten Gleichungssystems müssen noch zusätzliche Bedingungen eingeführt werden. Horn und Schunk [1] schlagen eine zusätzliche Glattheitsbedingung vor:

$$
\left(\frac{\partial u}{\partial x}\right)^{2}+\left(\frac{\partial u}{\partial y}\right)^{2}+\left(\frac{\partial v}{\partial x}\right)^{2}+\left(\frac{\partial v}{\partial y}\right)^{2}=\min
$$

Dadurch werden kontinuierliche Übergänge im Bewegungsvektorfeld erzwungen, was in den meisten Fällen günstigere Ergebnisse liefert als die isolierte Bestimmung von Bewegungsvektoren. Diese Bedingungen können zu einem Fehlerkriterium bzw. einer Kostenfunktion zusammengefaßt werden. Die Minimierung dieses Fehlerkriteriums führt zu den gesuchten Bewegungsvektoren. Der Optische Fluß liefert so für das gesamte Bild ein Vektorfeld mit einem Verschiebungsvektor pro Bildpunkt.

In [3] wird gezeigt, daß Bewegungsschätzungen nach Horn und Schunk eine starke Verbreiterung der Vektor- felder zeigen, d.h. durch die Glattheitsbedingung werden an einigen Stellen Bewegungsvektoren erzeugt. obwohl dort keine Bewegung stattfindet. In den hier betrachteten Bildern tritt dies vor allem an den Rändern des Ventrikels und an den Rändern der Herzklappen auf. Dieser Effekt wird durch Maskierung basierend auf einer Segmentierung der Bilder unterdrückt. Die Segmentierung erfolgt mit der im folgenden erläuterten Skalierungs-Index-Methode (SIM)[2].

\section{SKALIERUNGS-INDEX-METHODE}

Die Segmentierung der Ultraschallbilder geschieht durch die Untersuchung der räumlichen Umgebung eines jeden Bildpunktes. Zunächst betrachtet man jeden Bildpunkt eines Grauwertbildes als Punkt in einem dreidimensionalen Raum $(x, y, I)$. Die Bildintensitäten I sind also in der dritten Dimension über den Ortskoordinaten $x, y$ aufgetragen. Um jeden Punkt werden Kugeln unterschiedlicher Radien gelegt. und die Anzahl $N$ der Bildpunkte innerhalb dieser Kugeln wird bestimmt. Der Zusammenhang zwischen Kugelradius $r$ und Anzahl der enthaltenen Bildpunkte $N(r)$ liefert damn eine Aussage über die Zugehörigkeit des Punktes zu seiner Umgebung. Die Kurve $N(r)$ wird logarithmiert und dann durch eine Gerade approximiert.

Die Steigung dieser Gerade definiert den SkalierungsIndex des gerade untersuchten Punktes.

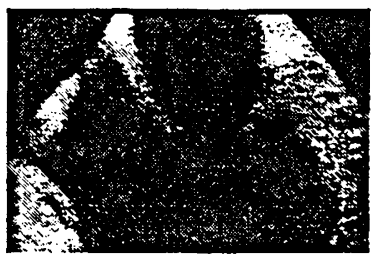

a) Ultraschallbild

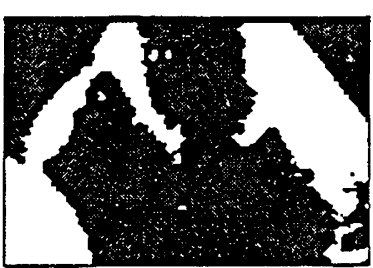

c) Segmentierung

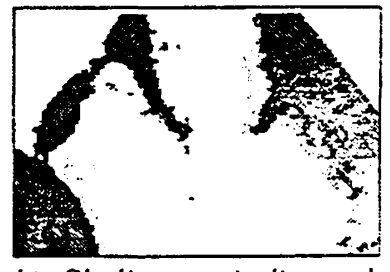

b) Skalierangsindizes als Grauwerte kodiert
Abbildung 1: Skalierungs-Index und Segmentierung

Die Verteilung der Indizes aller Bildpunkte liefert ein Histogramm, auf dessen Basis eine Schwelle zur Segmentierung des Bildes definiert werden kann. Das 
Ultraschallbild des menschlichen Herzens wird so in Bereiche des Ventrikels und des Myokards unterteilt. Abbildung 1 zeigt ein Ultraschallbild, die daraus berechneten Skalierungsindizes und die abgeleitete Segmentierungsmaske.

\section{UlTRASCHALL-BEWEGUNGSSCHÄTZUNG}

Ziel der hier dargestellten Arbeiten ist eine Bewegungsschätzung anhand von Ultraschallbildern des menschlichen Herzens. Es werden zwei Bilder einer Ultraschall-Bildfolge mit dem Ziel der Bewegungsdetektion untersucht (Abbildung 2). Die Bilder zeigen die sich öffnenden Herzklappen des linken Ventrikels des menschlichen Herzens. Eine Bewegungsschätzung kann hier einer verbesserten Diagnostik dienen. Die folgende Abbildung zeigt die beiden untersuchten Ultraschallbilder (Abbildung 2a,b)).
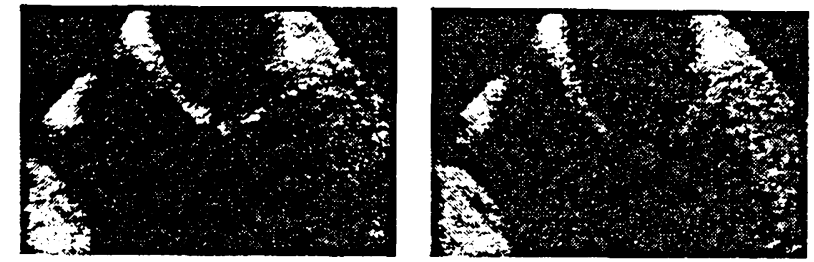

a) Originalbild 1

b) Originalbild 2

\section{Abbildung 2: Ultraschallbilder}

\section{ERGEBNISSE}

In den Abbildungen 3 und 4 sind die Ergebnisse der Bewegungsschätzung mittels optischem Fluß dargestellt. Abbildung 3 zeigt das Vektorfeld im Bereich der Herzklappen ohne Segmentierung, Abbildung 4 das Ergebnis der Berechnungen mit der in Abbildung (c) angegebenen Maske. Das nach der Berechnung des Optischen Flusses vorliegende Vektorfeld (Abbildung 3) beschreibt zwar die Bewegungen in den interessierenden Bildbereichen gut. insbesondere an Grenzflächen kommt es jedoch zu der in [3] beschriebenen Ausweitung des Vektorfeldes, was eine Interpretation der Ergebnisse erschwert.

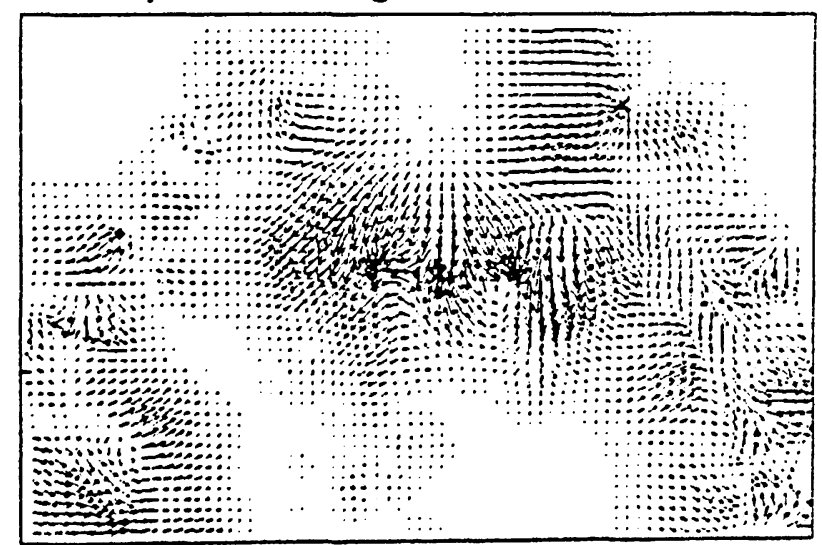

Abbildung 3: Vektorfeld der Herzklappen ohne Maskierung

Eine Maskierung auf Basis einer guten Segmentierung kann die Zuordnung der Vektoren zu den Bereichen des Myokards deutlich verbessern. In dem in Abbildung 4 dargestellten Vektorfeld wird so insbesondere die Richtung und der Betrag der Verschiebung an den interessierenden Punkten verdeutlicht.

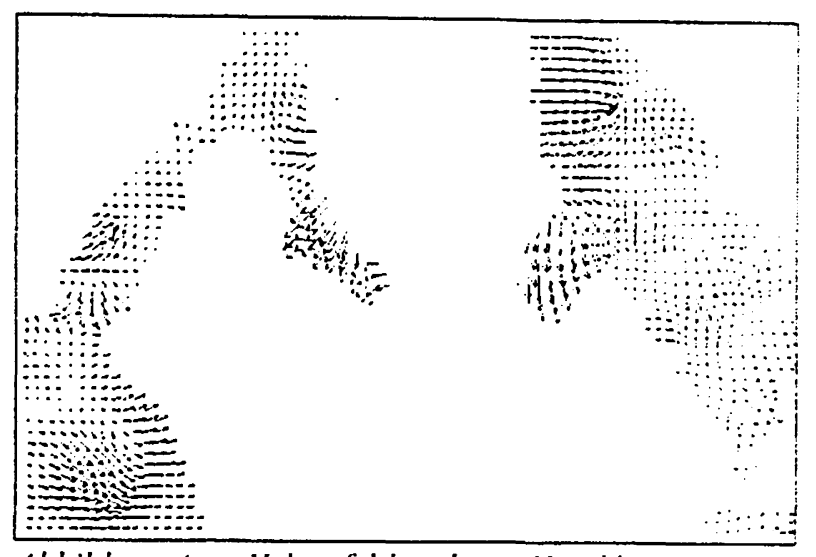

Abbildung 4: Veklorfeld der Her=kluppen mir Maskierung

Das folgende Diagramm faßt den Ablauf der Berechnungen graphisch zusammen.

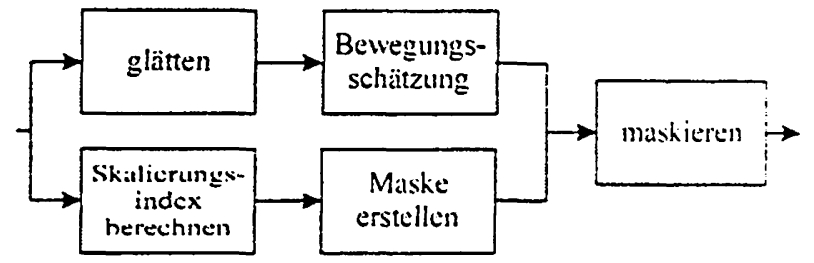

Abbildung 5: Verlauf der Berechmungen

Das Bewegungsvektorfeld. das mittels optischem Fluß3 berechnet wurde, wird mit einer Segmentierungsmaske verknüpft, die aus den Skalierungsindizes der Ultraschallbilder bestimmt wurde. Es findet also eine selektive Darstellung der berechneten Bewegungsvektoren statt.

\section{Zusammenfassung}

Der Optische Fluß ermöglicht eine gute Bewegungsschätzung in Ultraschallbildern des menschlichen? Herzens. Allerdings kommt es zu einer Ausweitung des Bewegungsvektorfeldes über die interessierenden Bereiche hinaus. was die Interpretation der Ergebnisse erschwert. Eine Segmentierung der relevanten Bildbereiche auf Basis der Skalierungs-Index-Methode erzielt eine deutliche Verbesserung der Bildqualität und ermöglicht so eine bessere Bewertung der Bewegungen des untersuchten Ultraschallbildes.

\section{LITERATUR}

[1] Horn, B. K. P.; Schunk B. G.: Determining opticul flow. Artificial Intelligence, 17, pp.185-204. 1981.

[2] Wagner, B.: Rauschen verboten. c't 11:1997. S. $318-322$.

[3] Baraldi, P. et. al.: Evaluation of Differcentiat Optical Flow Techniques on Symthesized Eilow Images: IEEE Transactions on Biomedical Engineering. Vol 43. No. 3. pp.259-271. 1996.

\section{Danksagung}

Die hier vorgestellten Arbeiten sind im Rahmen des ron der TU Berlin unterstutzten interdisziplinären Forschungsprojektes: ..Parallelrechnergestüzte Bewsgungsanalyse mit künstlichen Neurnolen N'tis:i" durchgefuhrt worden. 